\title{
G-protein subunit $\alpha-14$ is dysregulated in human placentas from systemic lupus erythematosus pregnancies
}

\author{
Ying-Jie Zhao ${ }^{1,2}$, Shan Tian ${ }^{3}$, Jia-Hui Xu' ${ }^{4}$ Colman I. Freel ${ }^{5}$, Kai Wang ${ }^{6}$, Huihui Li ${ }^{7}$
}

\author{
${ }^{1}$ Department of Rheumatology, Qilu Hospital, Cheeloo College of Medicine, Shandong \\ University, Jinan, Shandong, China \\ ${ }^{2}$ Shandong Provincial Clinical Research Center for Immune Diseases and Gout, Jinan, \\ Shandong, China \\ ${ }^{3}$ Center for Reproductive Medicine, Jinan Central Hospital, Jinan, Shandong, China \\ ${ }^{4}$ School for Radiological and Interdisciplinary Sciences, Soochow University, Suzhou, \\ Jiangsu, China \\ ${ }^{5}$ Department of Obstetrics and Gynecology, School of Medicine and Public Health, \\ University of Wisconsin-Madison, Madison, WI, USA \\ ${ }^{6}$ Clinical and Translational Research Center, Shanghai First Maternity and Infant \\ Hospital, Tongji University School of Medicine, Shanghai, China \\ ${ }^{7}$ Department of Obstetrics and Gynecology, Qilu Hospital, Cheeloo College \\ of Medicine, Shandong University, Jinan, Shandong, China
}

Submitted: 14 September 2021; Accepted: 3 February 2022

Online publication: 18 February 2022

Arch Med Sci

DOI: https://doi.org/10.5114/aoms/146404

Copyright $\odot 2022$ Termedia \& Banach

\begin{abstract}
Introduction: Systemic lupus erythematosus (SLE) is associated with placental dysfunctions during pregnancy, which may cause multiple adverse fetal and maternal outcomes. G-protein subunits $\alpha-11$ (GNA11) and -14 (GNA14) participate actively in angiogenesis via the modulation of endothelial function. This study aimed to determine whether GNA11 and GNA14 levels in placental tissues differed between SLE and normal term pregnancies.

Material and methods: Twenty-four individuals, including 14 patients with SLE and 10 normal pregnant women, were included in this study. The expression levels of GNA11 and GNA14 in the placentas were examined using reverse transcription-quantitative polymerase chain reaction (RT-qPCR) and Western blotting analyses. The localization of GNA11 and GNA14 in placental tissues was evaluated using immunohistochemistry. The correlations between relative mRNA and protein expression levels of GNA11,14 and the Systemic Lupus Erythematosus Disease Activity Index (SLEDAI) scores were also analyzed.

Results: RT-qPCR revealed that the mRNA levels of GNA14, but not GNA11, were significantly decreased by $50 \%(p=0.01)$ in SLE vs. normal term placentas. Western blotting showed that the protein levels of GNA14, but not GNA11, were significantly increased by 3.52 -fold $(p=0.02)$ in SLE vs. normal term placentas. In immunohistochemistry, positive staining for GNA11 and GNA14 was observed in trophoblasts and villous stromal cells of the placental tissues. The expression levels of GNA11 and GNA14 were not significantly correlated with the SLEDAI scores $\left(r^{2}=0.02 \sim 0.24, p=0.08 \sim 0.75\right)$.
\end{abstract}

Conclusions: The dysregulation of GNA14 in the placentas indicates a regulatory role during human SLE pregnancies.

Key words: systemic lupus erythematosus, G-protein, placenta, pregnancy.

\author{
Corresponding author: \\ Huihui Li PhD \\ Department of Obstetrics \\ and Gynecology \\ Qilu Hospital \\ Cheeloo College of Medicine \\ Shandong University \\ Jinan 250012, Shandong, \\ China \\ E-mail: hhli_qilu@email.sdu. \\ edu.cn
}




\section{Introduction}

Systemic lupus erythematosus (SLE) pregnancies are associated with several placental changes, including decreased placental weight, intraplacental hematoma, chronic villitis, ischemic hypoxic change, and placental infarction [1], which may result in adverse fetal outcomes, including preterm birth, stillbirth, spontaneous abortion, fetal growth restriction, and neonatal lupus [2, 3]. Additionally, maternal complications during SLE pregnancies include hypertension, preeclampsia, eclampsia, and lupus flare $[2,3]$.

G protein $\alpha$ subunit (GNA) 11 and GNA14, members of the $\mathrm{G} \alpha q / 11$ subfamily of the $G$ protein $\alpha$ subunit, have been shown to localize in different cell types of multiple human tissues, including endothelial and smooth muscle cells from lung tissues, endothelial and trophoblast cells from placentas, and tumor tissues [4-6]. Experimental data have demonstrated the essential roles of GNA11 and GNA14 in fibroblast growth factor 2 (FGF2)- and vascular endothelial growth factor (VEGF)- mediated fetoplacental endothelial cell functions and angiogenesis [7-9].

In this study, we aimed to investigate the expression of GNA11 and GNA14 in human placental tissues obtained from normal term and SLE pregnancies. The correlations between expression levels of GNA11,14 and the Systemic Lupus Erythematosus Disease Activity Index (SLEDAI) scores were also analyzed.

\section{Material and methods}

\section{Collection of placental tissues}

The study was conducted in accordance with the 1964 Helsinki Declaration. Placental tissues from normal term $(n=10)$ pregnancies were collected in Shanghai First Maternity and Infant Hospital affiliated with Tongji University. Tissue collection was approved by the Ethical Committee of Shanghai First Maternity and Infant Hospital affiliated with Tongji University (Ethical approval number: KS2013). Placental tissues from SLE $(n=14)$ pregnancies were collected in Qilu Hospital, Shandong University. Tissue collection was approved by the Institutional Review Board of Qilu Hospital, Shandong University (Ethical approval number: KYLL-2018-216). The American College of Rheumatology classification criteria were used to diagnose SLE [10]. The SLE disease activity index (SLEDAI) [11] was used to assess the disease activity of SLE patients. Informed consent was obtained from all participants. Smokers and participants with diabetes mellitus or cancer were excluded. Patient characteristics are described in Table I.

Placental tissues were obtained within $30 \mathrm{~min}$ after vaginal delivery or cesarean section delivery. Placental villi were dissected from beneath the chorionic and basal plates $(\sim 1 \times 1 \mathrm{~cm})$. After removal of the chorionic plate and amnion, chorionic tissues were snap-frozen in liquid nitrogen and stored at $-80^{\circ} \mathrm{C}$. Additional tissues were fixed

Table I. Clinical and laboratory characteristics

\begin{tabular}{|lccc|}
\hline Characteristics & SLE $(n=14)$ & Normal term $(n=10)$ & $P$-value \\
\hline Age [years], median (range) & $29.0(22-36)$ & $30.5(28-33)$ & 0.40 \\
\hline BMI $\left[\mathrm{kg} / \mathrm{m}^{2}\right.$ ], median (range) & $24.9(22.4-32.3)$ & $28.0(21.8-32.7)$ & 0.29 \\
\hline Vaginal delivery, $n$ (\%) & $8(57.1)$ & $6(60)$ & 0.89 \\
\hline Gestational age [weeks], median (range) & $38.4(34.9-39.7)$ & $39.1(38.6-40.1)$ & 0.02 \\
\hline Fetal weight [g], median (range) & $2950.0(2150.0-3850.0)$ & $3402.5(2895.0-3730.0)$ & 0.01 \\
\hline Disease duration [months], median (range) & $46.5(10-167)$ & - & - \\
\hline SLEDAl score, median (range) & $2(0-6)$ & - & - \\
\hline ANA $>1: 320$, yes/no, $n$ & $14 / 0$ & - & - \\
\hline Anti-dsDNA, yes/no, $n$ & $2 / 12$ & - & - \\
\hline Anti-phospholipid, yes/no, $n$ & $4 / 10$ & - & - \\
\hline Preeclampsia, yes/no, $n$ & $0 / 14$ & - & - \\
\hline Proteinuria, yes/no, $n$ & $3 / 11$ & - & - \\
\hline Hypocomplementemia, yes/no, $n$ & $7 / 7$ & - & - \\
\hline Steroids, yes/no, $n$ & $14 / 0$ & - & - \\
\hline Immunosuppressive drugs, yes/no, $n$ & $0 / 14$ & - & - \\
\hline Aspirin, yes/no, $n$ & $4 / 10$ & & - \\
\hline SLE - systemic lupus erythematosus, BMI - body mass index, SLEDAl - Systemic Lupus Erythematosus Disease & Activity Index, \\
ANA - antinuclear antibody. & & & - \\
& & - & - \\
\hline
\end{tabular}


Table II. Reverse transcription-quantitative polymerase chain reaction (RT-qPCR) primers

\begin{tabular}{|lccc|}
\hline Gene ID & Gene name & Forward $\left(5^{\prime}\right.$ - $\mathbf{3}^{\prime}$ ) & Reverse (5' - $^{\prime}$ ) \\
\hline ENSG00000111640 & GAPDH & TGACTTCAACAGCGACACCCA & CACCCTGTTGCTGTAGCCAAA \\
\hline ENSG00000088256 & GNA11 & GCATGTGGGGTGTGTGTTT & ATGGTCGGAGAAGGTGGTC \\
\hline ENSG00000156049 & GNA14 & GCCCACTCCTCCCCTATAA & GGCTGGCTCTGGTGATGT \\
\hline
\end{tabular}

in $4 \%$ paraformaldehyde solution in PBS at $4^{\circ} \mathrm{C}$ overnight and embedded in paraffin before being sectioned for immunohistochemistry.

\section{Reverse transcription-quantitative polymerase chain reaction (RT-qPCR)}

Total RNA was extracted using the RNeasy kit (Qiagen, cat\#: 74004). RNA concentration was measured using a Nanodrop One spectrophotometer (Thermo Fisher Scientific). RNAs were transcribed into complementary DNA using the HiScript II Q RT SuperMix for qPCR (Vazyme Biotech, cat\#: R222-01). RT-qPCR ( $=8$ /group) reactions were carried out using NuHi Robustic SYBR Green Mix (Nuhigh Biotechnologies, cat\#: NH9211) on an ABI 7900 Sequence Detection System. The primer sequences are listed in Table II. The PCR protocol was: $95^{\circ} \mathrm{C}$ for $10 \mathrm{~min}$, 40 cycles of $95^{\circ} \mathrm{C}$ for $15 \mathrm{~s}$, and $60^{\circ} \mathrm{C}$ for $60 \mathrm{~s}$. Melting curve analysis was used to confirm the amplification specificity. Data were normalized to GAPDH $[12,13]$. The relative expression levels of GNA11 and GNA14 were analyzed using the $2^{-\triangle \Delta C T}$ method $[14,15]$.

\section{Western blotting}

Western blotting was performed as described $[16,17]$. Placental tissues were homogenized on ice and lysed in lysis buffer (Cell Signaling, cat\#: 9803) by sonication. Supernatants were obtained after centrifugation at $4^{\circ} \mathrm{C}$ for $20 \mathrm{~min}(12,000 \mathrm{~g})$. Protein samples $(100 \mu \mathrm{g})$ were added to the loading buffer and heated at $95^{\circ} \mathrm{C}$ for $5 \mathrm{~min}$ to denature. Protein samples ( $n=10$ for normal term group and 14 for SLE group) were separated on $10 \%$ SDS-PAGE gel and transferred to polyvinylidene difluoride membranes. Tris-buffered saline was used to prepare solutions including blocking buffer and antibody solutions. After blocking with 5\% skim milk in TBS buffer for $1 \mathrm{~h}$, the membranes were probed with GNA11 (1:1000, Abgent, cat\#: AP19441a) or GNA14 antibody (1:500, Abnova, cat\#: H00009630-M06A) at $4^{\circ} \mathrm{C}$ overnight, followed by reprobing with GAPDH (1:10,000; Abnova, cat\#: H00002597-M01) as controls. Secondary antibodies were incubated with membranes at room temperature for 1 h. Protein immunoreactivity was visualized with ECL2 or ECL reagent (Thermo Fisher Scientific, cat\#: 80196; 32106). Densitometry data obtained by the NIH Image-J imaging analysis software (Bethesda, Maryland, USA) were used to quantify the protein expression levels of GNA11 and GNA14.

\section{Immunohistochemistry}

Immunohistochemistry was performed as previously described [18, 19]. Paraffin-embedded placental tissues ( $n=4$ /group) were sliced into $4 \mu \mathrm{m}$ thickness sections. Antigen retrieval was performed in citrate acid solution $(10 \mathrm{mM}$ citrate acid, $\mathrm{PH}$ 6.5) and boiled for $10 \mathrm{~min}$. Endogenous peroxidase was quenched using $3 \%$ hydrogen peroxide at room temperature for $10 \mathrm{~min}$. After being blocked with blocking serum at room temperature for $30 \mathrm{~min}$, tissue sections were probed with primary antibodies diluted in $10 \mathrm{mM} \mathrm{PBS} / 0.3 \%$ Triton-X100 against GNA11 (4 $\mathrm{gg} / \mathrm{ml}$, Abgent, cat\#: AP19441a) or GNA14 (4 $\mathrm{\mu g} / \mathrm{ml}$, Abnova, cat\#: H00009630-M06A) at $4^{\circ} \mathrm{C}$ overnight and then incubated with a biotinylated universal secondary antibody (Vector Laboratories, BP-1400-50) at room temperature for $30 \mathrm{~min}$. The staining was visualized with the AEC Substrate Kit (Vector Laboratories, cat\#: SK-4200) at room temperature for up to $20 \mathrm{~min}$. Brownish-yellow staining was considered as giving a positive signal. Finally, tissue sections were re-stained with hematoxylin for $5 \mathrm{~min}$.

\section{Correlation analysis of GNA11, 14 levels and SLEDAI scores}

According to RT-qPCR and Western blotting data, relative expression levels of GNA11 and GNA14 (fold of normal term) were used to analyze correlation with SLEDAI scores.

\section{Statistical analysis}

Data were expressed as median \pm standard deviation or median with range. Data were analyzed using the Mann-Whitney rank sum test or Student's t-test when applicable. The correlation analysis was performed using Pearson's correlation coefficient. $P$-values $<0.05$ were considered statistically significant. Sample size calculation (power analysis) was analyzed according to the study of Rosner B [20]. The statistical results in the current study are reliable based on the sample size that we used. SigmaPlot (13.0) for Windows was used for statistical analyses. 


\section{Results}

All SLE patients received maintenance corticosteroids (prednisone $\leq 15 \mathrm{mg}$ daily) and hydroxychloroquine ( $\leq 400 \mathrm{mg}$ daily) during pregnancy. The SLEDAI scores prior to delivery ranged from 0 to 6 . None of the newborns developed neonatal lupus. Maternal age and body mass index (BMI) were similar between normal term and SLE. Gestational ages for SLE pregnancies (38.4 \pm 1.3 weeks) were lower $(p=0.02)$ than in normal term pregnancies (39.1 \pm 0.6 weeks). Fetal weights for SLE pregnancies (2950.0 $\pm 442.1 \mathrm{~g})$ were lower $(p=0.01)$ than in normal term pregnancies (3402.5 $\pm 262.0 \mathrm{~g})$. Six patients in the normal term and eight in the SLE group underwent vaginal delivery, without significantly different distribution between two groups.

RT-qPCR was conducted to examine GNA11 and GNA14 mRNA relative expression levels in SLE and normal term placentas. The mRNA levels of GNA14, but not GNA11, were significantly decreased by $50 \%(p=0.01)$ in SLE vs. normal term placentas.

Western blotting analysis showed that GNA11 and GNA14 proteins were detected at $\sim 42 \mathrm{kDa}$ (Figure 1). The protein levels of GNA14, but not GNA11, were significantly increased 3.52-fold ( $p=$ 0.02 ) in SLE vs. normal term placentas when normalized to GAPDH (Figure 1).

Immunohistochemistry was conducted to examine the localization of GNA11 and GNA14 in SLE and normal term placentas. GNA11 and GNA14 were localized in trophoblasts and villous stromal cells in SLE and normal term placentas (Figure 2).
We further analyzed the correlation between expression levels of GNA11, 14, and SLEDAI scores of SLE patients. The relative $\mathrm{mRNA}$ and protein expression levels of GNA11 and GNA14 were not significantly correlated with the SLEDAI scores $\left(r^{2}=0.02 \sim 0.24, p=0.08 \sim 0.75\right.$; Figure 3$)$.

\section{Discussion}

Our study demonstrates the localization and expression levels of GNA11 and GNA14 in human placentas from normal term and SLE pregnancies. We observed that GNA11 and GNA14 are localized in different cell types in human placental tissues, including trophoblasts and villous stromal cells. Our findings agree with a previous study concerning umbilical cord and placental tissues from preeclampsia patients that GNA11 and GNA14 are present in endothelial cells, trophoblasts, and villous stromal cells [5]. More importantly, we have demonstrated that GNA14, and not GNA11, is downregulated in SLE vs. normal term at mRNA levels in placentas; however, GNA14, and not GNA11, is upregulated in SLE vs. normal term at protein levels. These data suggest that GNA11 and GNA14 may be associated with placental cell functions, and GNA14 may play a unique role in regulating placental functions during SLE pregnancies.

Downregulation of GNA14 at mRNA levels and upregulation at protein levels indicate a discordant expression between mRNA and protein levels from SLE vs. normal term placentas. Studies investigating the correlations between mRNA and protein abundance in human tissues are contro-
A

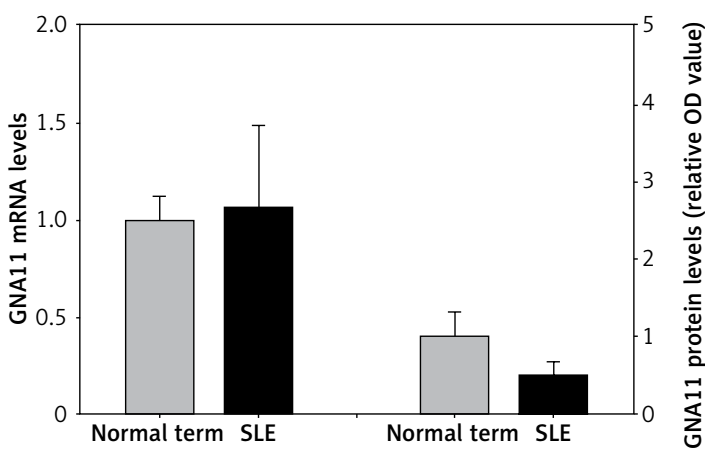

B

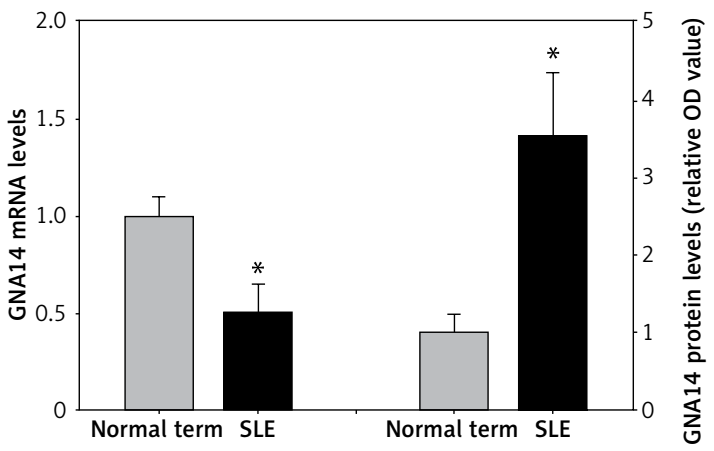

C

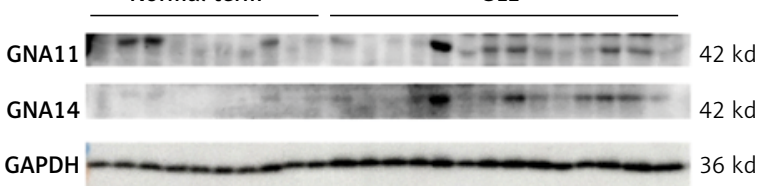

Figure 1. GNA11 and GNA14 expression patterns in human placentas. A, B - RT-qPCR and Western blotting analyses. C - Representative Western blotting for the GNA and GNA14 protein levels (100 $\mu$ g of each placental sample) in human placentas from systemic lupus erythematosus (SLE) and normal term pregnancies $(n=10 \sim 14 /$ group) ${ }^{*} p<0.05$ 
GNA11
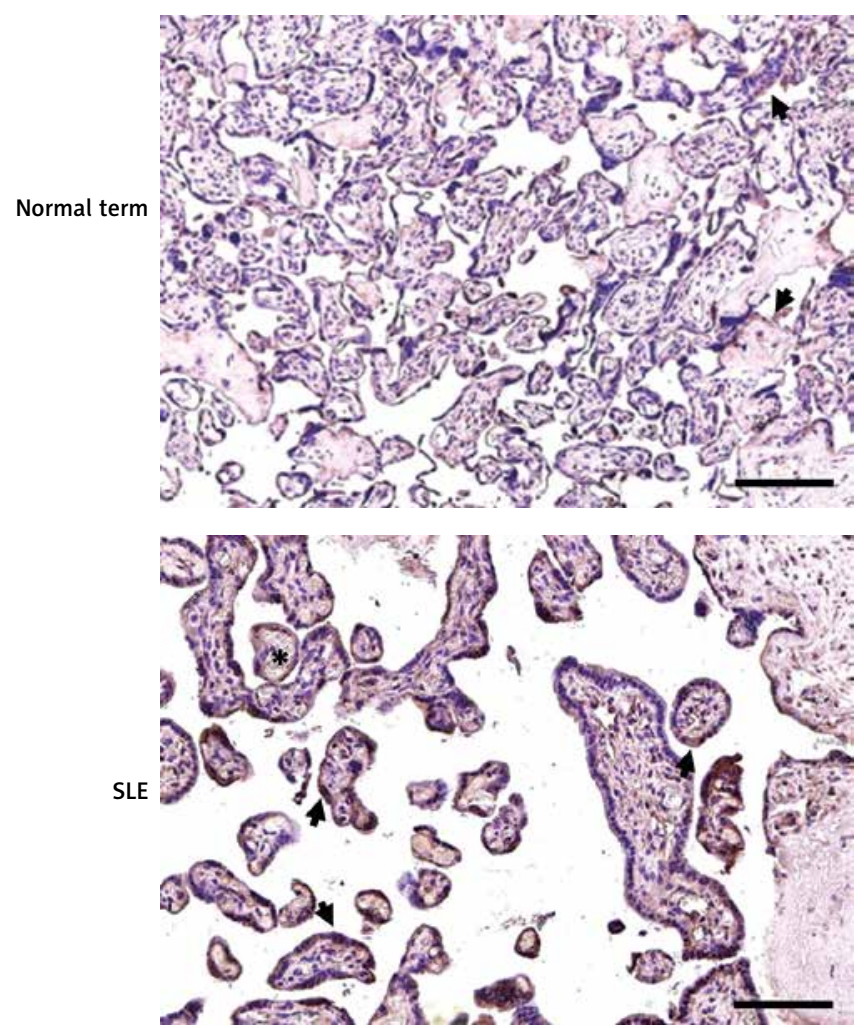

GNA14
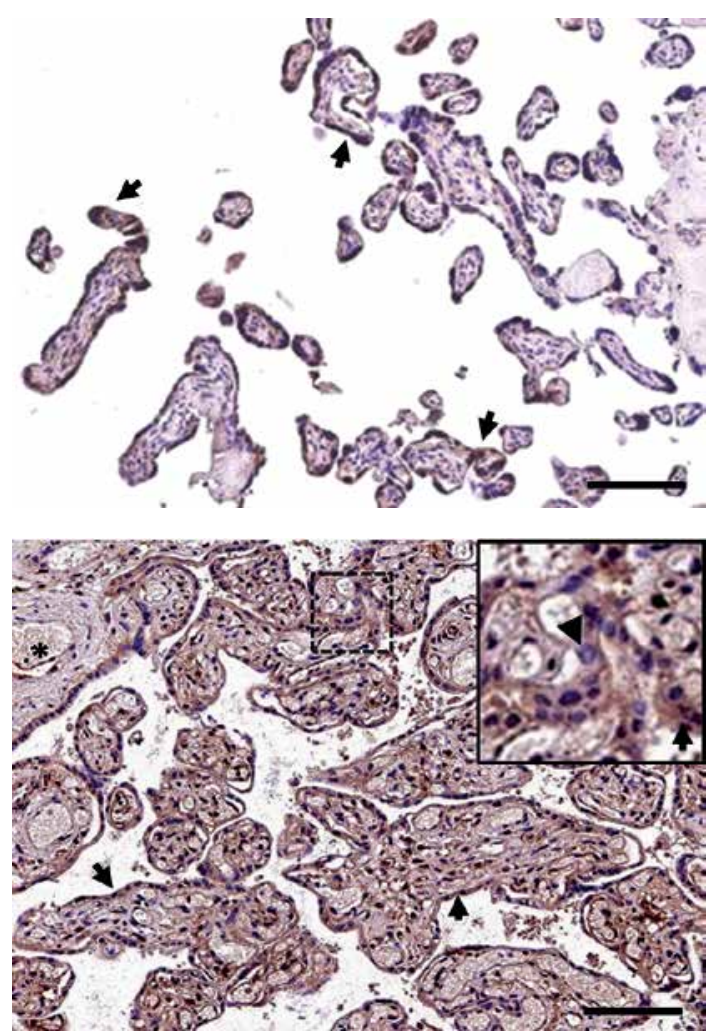

Figure 2. Immunolocalization of GNA11 and GNA14 in human placentas from systemic lupus erythematosus (SLE) and normal term pregnancies

*lumen of blood vessels, arrows - syncytiotrophoblast, arrowheads - cytotrophoblast

versial [21-23]. A study involving 23 human cell lines showed that correlations were only detected in one-third of the mRNA and corresponding proteins [24]. In this study, two different delivery methods, vaginal birth and C-section, were performed, which may have different impacts on gene expression. However, there was no significantly different distribution between the two groups (vaginal delivery [\%] from SLE group $=57.1 \%$ vs. $60 \%$ from the normal term group, $p=0.89$ ). Subgroup RT-qPCR analysis showed no significant difference in mRNA expression levels of vaginal birth and C-section for both GNA11 and GNA14 in both groups (Supplementary Figure S1, $p=0.28 \sim 0.44$ ). Further studies involving more patients are required to confirm this finding. Several factors may contribute to the absence of a strong correlation between mRNA and protein levels. First, post-transcriptional and/or post-translational mechanisms are complicated and varied among different genes [21]; second, proteins have different half-lives [25]; third, there are error and noise in mRNA and protein experiments [21]; additionally, alternative methods of examination may result in different data with varied correlations. The above reasons may contribute to the discordant expression levels of GNA14 between mRNA and protein levels from SLE placentas in this study.
GNA11 and GNA14 have been demonstrated to play essential roles in fetoplacental endothelial cell functions and angiogenesis [7-9]. The current finding that GNA14, but not GNA11, is dysregulated in SLE placentas implies that GNA14 may be a unique mediator in placentas from SLE pregnancies, in which dysfunction of angiogenesis may be involved. Higher levels of angiogenic activity have been reported in serum samples from patients with SLE, which is associated with SLE disease activity [26]. Our bioinformatics data concerning differential expressed genes of placentas between SLE and normal control pregnancy also showed that angiogenesis (Gene Ontology term: GO:0001525) was enriched (data not shown), suggesting that dysfunction of angiogenesis may be associated with placental development during SLE pregnancies. The dysregulation of GNA14 in the placentas suggests its regulatory role in placental angiogenesis during SLE pregnancies, which may lead to dysfunction of the placentas [27]. However, the function of GNA14 in SLE placental cells remains unclear. Further functional studies are warranted to explore the potential mechanisms of GNA14 in the angiogenesis function of SLE placentas.

It remains elusive what causes the dysregulation of GNA14 in SLE placentas. In the current study, gestational ages and fetal weights in the 
A

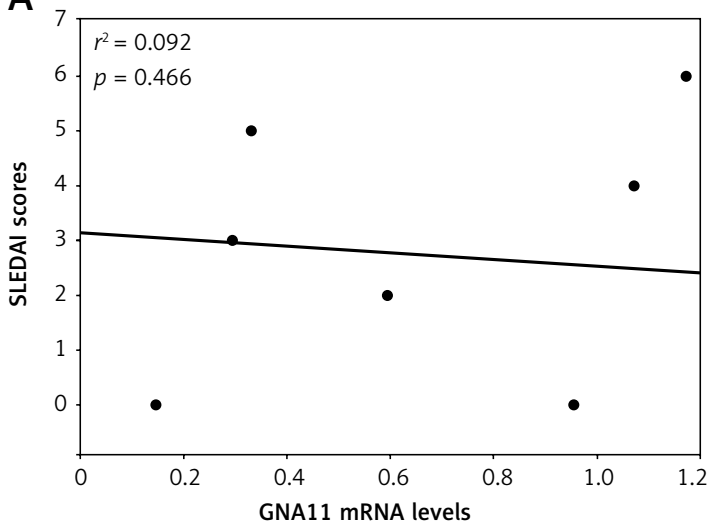

C

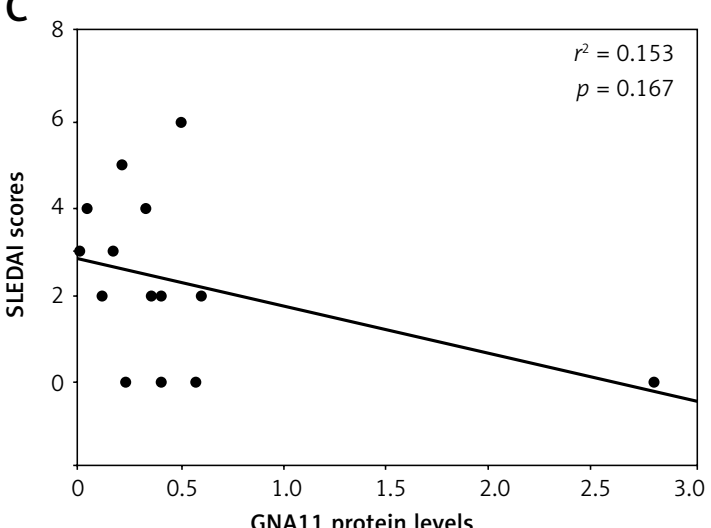

B

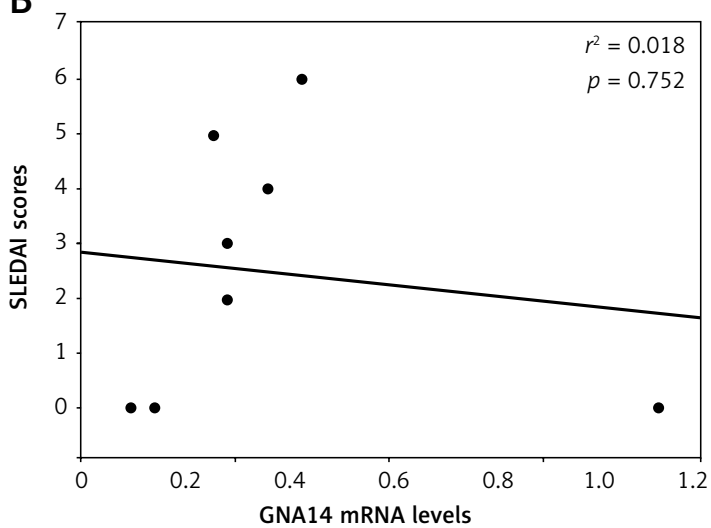

D

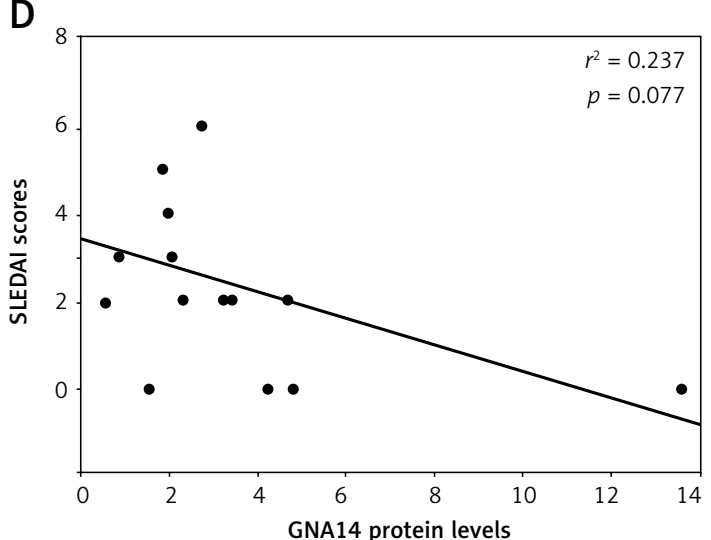

Figure 3. Correlation analyses between GNA11, 14 expression levels and SLEDAI scores. Relative expression levels of GNA11 and GNA14 were obtained using RT-qPCR and Western blotting analyses. Correlations between SLEDAl scores and expression levels of GNA11 and GNA14 (fold of the normal term) were analyzed using Pearson's correlation coefficient

SLE group were lower than in the controls, which is in line with the evidence that SLE pregnancies are associated with adverse pregnancy outcomes, including preterm birth and fetal growth restriction $[2,3]$. The aberrant expression of GNA14 in placentas may attribute to different gestation ages and fetal weights between SLE and normal term pregnancies. New reliable clinical biomarkers may provide novel therapies for SLE. The dysregulation of GNA14 in SLE placentas suggests that GNA14 might become a potential biomarker and therapeutic target for SLE. However, the correlation analysis in this study did not show a statistically significant association between SLEDAI scores and GNA14 expression levels in SLE placentas. A small case number may contribute to the above finding. Meanwhile, SLEDAI scores of SLE patients in this study ranged from 0 to 6 , and the limited SLEDAI score range may limit the correlation analysis. Further studies involving a larger patient number and wider SLEDAI score range are necessary to investigate the potential roles of GNA14 during SLE pregnancies.

To our knowledge, this is the first report investigating the mRNA and protein expression levels of GNA11 and GNA14 in placental tissues from
SLE pregnancies. The current results suggest that GNA11 and GNA14 may participate in placental cellular functions. The dysregulation of GNA14 in the SLE placentas suggests that GNA14 may contribute to the dysfunction of placental cells during SLE pregnancies, and GNA14 might serve as a potential biomarker and therapeutic target for SLE. Meanwhile, there are several limitations to this study. First, we were limited by the small sample size. Second, low disease activity (SLEDAI scores from 0-6) of SLE patients included in this study restrained us from exploring the relationship between GNA11 and GNA14 levels with patients with higher SLEDAl scores. Further studies are necessary to define the potential roles of GNA11 and GNA14 during SLE pregnancies.

In conclusion, the current data provide evidence that GNA14 is dysregulated in human SLE placentas. The dysregulation of GNA14 in the placentas indicates a regulatory role during SLE pregnancies.

\section{Acknowledgments}

Ying-Jie Zhao and Shan Tian contributed equally to this work. 
The study was supported by Shandong provincial key research and development program 2018GSF118071 (YJZ), 2018GSF118025 (HHL), and Shandong University Clinical Research Center Key Special Funds for emergency and critical diseases (2021SDUCRCB010).

\section{Conflict of interest}

The authors declare no conflict of interest.

\section{References}

1. Marder W, Knight JS, Kaplan MJ, et al. Placental histology and neutrophil extracellular traps in lupus and preeclampsia pregnancies. Lupus Sci Med 2016; 3: e000134.

2. Baer AN, Witter FR, Petri M. Lupus and pregnancy. Obstet Gynecol Surv 2011; 66: 639-53.

3. Smyth A, Oliveira GH, Lahr BD, Bailey KR, Norby SM, Garovic VD. A systematic review and meta-analysis of pregnancy outcomes in patients with systemic lupus erythematosus and lupus nephritis. Clin J Am Soc Nephrol 2010; 5: 2060-8.

4. Lei W, Chen P, Yue Y, et al. Subcellular distribution patterns and elevated expression of GNA11 and GNA14 proteins in the lungs of humans with pulmonary arterial hypertension. Cell Biol Int 2014; 38: 1041-9.

5. Zhao YJ, Zou QY, Li Y, et al. Expression of G-protein subunit $\alpha-14$ is increased in human placentas from preeclamptic pregnancies. J Histochem Cytochem 2014; 62: 347-54.

6. Van Raamsdonk CD, Griewank KG, Crosby MB, et al. Mutations in GNA11 in uveal melanoma. N Engl J Med 2010; 363: 2191-9.

7. Zou QY, Zhao YJ, Li H, et al. GNA11 differentially mediates fibroblast growth factor 2 - and vascular endothelial growth factor A-induced cellular responses in human fetoplacental endothelial cells. J Physiol 2018; 596: 2333-44.

8. Zou QY, Zhao YJ, Zhou C, et al. G Protein $\alpha$ Subunit 14 Mediates Fibroblast Growth Factor 2-Induced Cellular Responses in Human Endothelial Cells. J Cell Physiol 2019; 234: 10184-95.

9. Liau JY, Lee JC, Tsai JH, Chen CC, Chung YC, Wang YH. High frequency of GNA14, GNAQ, and GNA11 mutations in cherry hemangioma: a histopathological and molecular study of 85 cases indicating GNA14 as the most commonly mutated gene in vascular neoplasms. Mod Pathol 2019; 32: 1657-65.

10. Petri M, Orbai AM, Alarcón GS, et al. Derivation and validation of the Systemic Lupus International Collaborating Clinics classification criteria for systemic lupus erythematosus. Arthritis Rheum 2012; 64: 2677-86.

11. Bombardier C, Gladman DD, Urowitz MB, Caron D, Chang $\mathrm{CH}$. Derivation of the SLEDAI. A disease activity index for lupus patients. The Committee on Prognosis Studies in SLE. Arthritis Rheum 1992; 35: 630-40.

12. Chen Z, Gao Y, Gao S, Song D, Feng Y. MiR-135b-5p promotes viability, proliferation, migration and invasion of gastric cancer cells by targeting Krüppel-like factor 4 (KLF4). Arch Med Sci 2020; 16: 167-76.

13. Bai $Y$, Chen $Y$, Chen $X$, et al. Trichostatin A activates FOXO1 and induces autophagy in osteosarcoma. Arch Med Sci 2019; 15: 204-13.
14. Li Y, Wang K, Zou Q-y, Jiang Y-z, Zhou C, Zheng J. ITE suppresses angiogenic responses in human artery and vein endothelial cells: differential roles of AhR. Reprod Toxicol 2017; 74: 181-8.

15. Zhou C, Yan Q, Zou QY, et al. Sexual dimorphisms of preeclampsia-dysregulated transcriptomic profiles and cell function in fetal endothelial cells. Hypertension 2019; 74: 154-63.

16. Chen Y, Bi Q, Zhu Z, et al. Lycium barbarum polysaccharides exert an antioxidative effect on rat chondrocytes by activating the nuclear factor (erythroid-derived 2)-like 2 signaling pathway. Arch Med Sci 2020; 16: 964-73.

17. Zhao M, Wang K, Shang J, Liang Z, Zheng W, Gu J. MiR$345-5 p$ inhibits tumorigenesis of papillary thyroid carcinoma by targeting SETD7. Arch Med Sci 2020; 16: 888-97.

18. Zhang G, Zha J, Liu J, Di J. Minocycline impedes mitochondrial-dependent cell death and stabilizes expression of hypoxia inducible factor- $1 \alpha$ in spinal cord injury. Arch Med Sci 2019; 15: 475-83.

19. Jin H, Zhang L, Wang S, Qian L. BST2 promotes growth and induces gefitinib resistance in oral squamous cell carcinoma via regulating the EGFR pathway. Arch Med Sci 2021; 17: 1772-82.

20. Rosner B. Fundamentals of Biostatistics. 7th ed. Brooks/ Cole, Cengage Learning, USA Boston 2011.

21. Yu EZ, Burba AE, Gerstein M. PARE: a tool for comparing protein abundance and mRNA expression data. BMC Bioinformatics 2007; 8: 309.

22. Pramatirta AY, Mose J, Effendi JS, et al. Correlation between cell-free mRNA expressions and PLGF protein level in severe preeclampsia. BMC Res Notes 2015; 8: 208.

23. Koussounadis A, Langdon SP, Um IH, Harrison DJ, Smith VA. Relationship between differentially expressed mRNA and mRNA-protein correlations in a xenograft model system. Sci Rep 2015; 5: 10775.

24. Gry M, Rimini R, Strömberg S, et al. Correlations between RNA and protein expression profiles in 23 human cell lines. BMC Genomics 2009; 10: 365.

25. Schwanhäusser B, Busse D, Li N, et al. Global quantification of mammalian gene expression control. Nature 2011; 473: 337-42.

26. Liu J, Wang X, Yang X, Yan Q, Wang S, Han W. Investigating the role of angiogenesis in systemic lupus erythematosus. Lupus 2015; 24: 621-7.

27. Gluhovschi C, Gluhovschi G, Petrica L, Velciov S, Gluhovschi A. Pregnancy associated with systemic lupus erythematosus: immune tolerance in pregnancy and its deficiency in systemic lupus erythematosus an immunological dilemma. J Immunol Res 2015; 2015: 241547. 